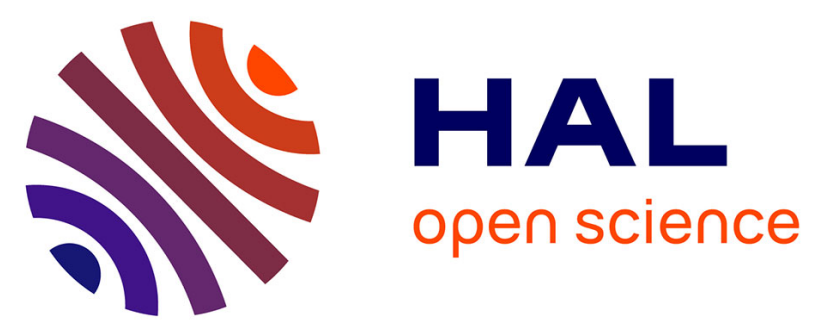

\title{
Surface plasmon resonance of an individual nano-object on an absorbing substrate: quantitative effects of distance and $3 \mathrm{D}$ orientation
}

\author{
Etienne Pertreux, Anna Lombardi, Ileana Florea, Miguel Spuch-Calvar, Sergio
}

Gómez-Graña, Dris Ihiawakrim, Charles Hirlimann, Ovidiu Ersen, Jérôme

Majimel, Mona Tréguer-Delapierre, et al.

\section{To cite this version:}

Etienne Pertreux, Anna Lombardi, Ileana Florea, Miguel Spuch-Calvar, Sergio Gómez-Graña, et al. Surface plasmon resonance of an individual nano-object on an absorbing substrate: quantitative effects of distance and 3D orientation. Advanced Optical Materials, 2016, 4 (4), pp.567-577. 10.1002/adom.201500548 . hal-01309285

\section{HAL Id: hal-01309285 https://hal.science/hal-01309285}

Submitted on 22 Jan 2021

HAL is a multi-disciplinary open access archive for the deposit and dissemination of scientific research documents, whether they are published or not. The documents may come from teaching and research institutions in France or abroad, or from public or private research centers.
L'archive ouverte pluridisciplinaire HAL, est destinée au dépôt et à la diffusion de documents scientifiques de niveau recherche, publiés ou non, émanant des établissements d'enseignement et de recherche français ou étrangers, des laboratoires publics ou privés. 


\title{
Surface Plasmon Resonance of an Individual Nano-Object on an Absorbing Substrate: Quantitative Effects of Distance and 3D Orientation
}

\author{
Etienne Pertreux, Anna Lombardi, Ileana Florea, Miguel Spuch-Calvar, \\ Sergio Gómez-Graña, Dris Ihiawakrim, Charles Hirlimann, Ovidiu Ersen, \\ Jérôme Majimel, Mona Tréguer-Delapierre, Mike Hettich, Paolo Maioli, \\ Aurélien Crut,* Fabrice Vallée, and Natalia Del Fatti
}

\begin{abstract}
Modification of the plasmonic response of a metal nano-object due to interaction with a substrate is experimentally investigated measuring the quantitative optical extinction spectra of individual nano-objects with various elongated shapes (bipyramids and rods) deposited on a dielectric (silica) or absorbing (carbon) membrane. Apart from the expected dependence of the nanoparticle surface plasmon resonance (SPR) frequency on the nature of the substrate, large substrate and particle shape dependent modifications of its SPR width are demonstrated. These dependencies are ascribed to strong localization of the electromagnetic field associated with the longitudinal SPR of an elongated nano-object around its tips, leading to different interaction with the substrate depending on the particle shape and 3D orientation relative to the substrate. Both parameters have been precisely determined by electron tomography, permitting excellent reproduction of the experimental data. Experiments performed on silver-encapsulated bipyramids, whose shape evolves from a pyramidal one towards a cylindrical one, further confirm this effect.
\end{abstract}

\section{Introduction}

The localized surface plasmon resonance (SPR) of metal nanoobjects is the key feature of their optical response. This and the local field enhancement it reflects have been extensively investigated during the last decade, both from experimental and

\footnotetext{
E. Pertreux, Dr. A. Lombardi, Dr. M. Hettich,

Dr. P. Maioli, Dr. A. Crut, Prof. F. Vallée,

Prof. N. Del Fatti

FemtoNanoOptics group

Institut Lumière Matière

CNRS-Université Lyon 1

Université de Lyon, France

E-mail: aurelien.crut@univ-lyon1.fr

Dr. I. Florea, D. Ihiawakrim, Prof. C. Hirlimann, Prof. O. Ersen

Institut de Physique et Chimie des Matériaux de Strasbourg

CNRS-Université de Strasbourg, France

Dr. M. Spuch-Calvar, Dr. S. Gómez-Graña, Dr. J. Majimel,

Dr. M. Tréguer-Delapierre

Institut de Chimie de la Matière Condensée de Bordeaux

CNRS-Université de Bordeaux, France
}

theoretical points of view. This interest has been primarily motivated by the possibility of tuning the optical properties of metal nano-objects, due to the large dependence of the SPR characteristics (spectral position, quality factor, amplitude, local electromagnetic field enhancement) on their shape and environment, ${ }^{[1,2]}$ and by the many applications they offer. The frequency dependence on the object local environment has for instance been exploited for biosensing applications or for realtime monitoring of chemical reactions, ${ }^{[3,4]}$ while electromagnetic fields enhancement around a nano-object associated to SPR has opened the possibility of label-free detection of molecules by enhancing their optical response (e.g., Raman scattering ${ }^{[5,6]}$ or fluorescence $\left.\mathrm{e}^{[7]}\right)$. Detailed understanding of the shape and environment effects on the SPR characteristics of a nano-object is thus of key interest. It can only be experimentally achieved on individual nano-objects, to avoid the spurious inhomogeneous effects due to unavoidable dispersion of the particle morphology in ensemble measurements. ${ }^{[2]}$ Many investigations have now been performed on single nanoparticles, most of them being deposited on a solid substrate, a situation also most frequently encountered in applications. ${ }^{[2,8,9]}$ Although good reproduction of experimental data has been obtained in the case of nano-objects deposited on a dielectric substrate, ${ }^{[10-15]}$ full and quantitative understanding of substrate impact is still lacking as it requires not only precise determination of the object morphology, but also of its position relative to the substrate (distance and orientation), especially for absorbing ones.

Interaction of an absorbing substrate with a deposited metal nanoparticle is still little studied. Though red shift and splitting of the SPR of single gold and silver particles on a gold film due to coupling with its propagating surface plasmon polaritons have been reported, ${ }^{[16-18]}$ a more specific effect of absorbing substrates is the induced broadening of the SPR. This has been observed for gold nano-objects lithographed on a chromium or titanium adhesion layer ${ }^{[19,20]}$ and gold nanorods standing on a graphene 
layer. ${ }^{[21]}$ The spatial distribution of the electromagnetic field and overlap of the zone where it is strongly enhanced with the substrate was pointed-out as a key parameter. ${ }^{[20]}$ This further stressed the importance of precise determination of the object morphology and position relative to the substrate when interpreting the optical response of single nano-objects spin-coated on a substrate, a common situation when they are chemically synthesized.

In this context elongated nano-objects are particularly interesting as their longitudinal SPRs are associated to localized enhancement of the electromagnetic field around their tips, with a spatial distribution strongly depending on their shape (e.g., rod as compared to bipyramid). ${ }^{[15]}$ We have quantitatively investigated the effect of an absorbing substrate on their plasmonic response measuring the absolute extinction spectra of individual gold nanorods and nanobipyramids deposited on thin dielectric (silica) and absorbing (carbon) substrates using spatial modulation spectroscopy (SMS), demonstrating larger substrate-induced SPR broadening for a nanorod than for a bipyramid. The morphology and position (distance and $3 \mathrm{D}$ orientation relative to the substrate) of the particles were determined using two- and three-dimensional transmission electron microscopy (TEM). ${ }^{[22]}$ These geometrical parameters were used in finite-element modeling (FEM) numerical simulations yielding excellent agreement with the experimental data, highlighting their impacts. These are further confirmed by extension of these experiments to silver-encapsulated gold nanobipyramids, which offer the unique possibility of changing their shape from a bipyramid to a rod one, and thus controlling their interaction with the substrate.

\section{Results and Discussion}

\subsection{Optical Experiments on Elongated Gold Nano-objects}

Solutions of surfactant-coated gold nanorods and nanobipyramids were synthesized using previously described procedures $^{[15,23]}$ (see Experimental Section for details about nanobipyramid synthesis). These colloidal solutions were spincoated on TEM grids covered by either $\mathrm{a} \approx 40 \mathrm{~nm}$ thick silica or $\mathrm{a} \approx 20 \mathrm{~nm}$ thick carbon membrane. The extinction crosssection $\sigma_{\text {ext }}$ of single nano-objects was quantitatively measured using $\operatorname{SMS}^{[2,24-26]}$ (Figure 1a, see also Experimental Section). The polarized spectra were fitted in the energy domain by a Lorentzian function, allowing extraction of the longitudinal SPR spectral position and width. ${ }^{[15]}$ TEM was used to individually correlate each measured extinction spectrum with the high-resolution image of the associated nano-object, allowing the determination of its dimensions (length $L$ and diameter $D$ ). Typical $\sigma_{\text {ext }}$ spectra of individual and isolated gold nanorods with lengths $L=35-45 \mathrm{~nm}$ and diameters $D=10-15 \mathrm{~nm}$ are shown in Figure 1b-d. The extinction spectra measured for deposition on silica and carbon membranes are compared for selected individual nanorods of same aspect ratio $(\eta=L / D$, Figure $1 b)$, same SPR position $\left(\lambda_{R}\right.$, Figure $\left.1 c\right)$ and same effective length characterizing quantum confinement-induced broadening effects $L_{e f f}=(S / \pi)^{1 / 2}$, where $S=\pi L D$ is the nanorod surface (Figure 1d). ${ }^{[27]}$ All the measured single particle SPR widths are plotted in Figure 1e as a function of this parameter.
As expected, red shifting of SPR was observed for nanorods of same aspect ratio deposited on carbon substrate as compared to silica one (Figure 1b). This $\Delta \lambda_{R} \approx 60 \mathrm{~nm}$ shift can be directly ascribed to a larger real part of its complex refractive index $\left(\operatorname{Re}\left(n_{s}\right)=2.3\right.$ in carbon, see below, as compared to $n_{s}=1.45$ for silica). In addition, nanorod deposition on carbon substrate leads to an important broadening of the longitudinal SPR as compared to a dielectric substrate (Figure 1b-e). On average, the SPR spectral width $\Gamma$ is two times larger on carbon $(\hbar \Gamma=(270 \pm 30) \mathrm{meV})$ than on silica $(\hbar \Gamma=(135 \pm 20) \mathrm{meV})$. This large broadening is observed independently of the parameter fixed for comparing single-nanorod spectra: $\eta$ (Figure 1b), $\lambda_{R}$ (Figure 1c) or $L_{\text {eff }}$ (Figure $1 \mathrm{~d}$ and $1 \mathrm{e}$ ).

The SPR width $\Gamma$ can be decompaosed as:

$\Gamma=\Gamma_{0}+\Gamma_{r}+\gamma_{s}+\Gamma_{c h e m}+\Gamma_{s u b}$

where $\Gamma_{0}, \Gamma_{r}, \gamma_{s}$ and $\Gamma_{\text {chem }}$ respectively describe the contributions of bulk-like Landau damping in the nano-object, radiative damping, quantum confinement and interface chemical damping effect (related to the presence of surfactant molecules), which have all been discussed in previous works, ${ }^{[2,27-33]}$ while $\Gamma_{\text {sub }}$ quantifies substrate effects (with $\Gamma_{\text {sub }}=0$ for a dielectric one), whose description is the primary scope of this paper. The $\Gamma$ values measured for surfactant-coated nanorods on silica substrate are larger by about $40 \mathrm{meV}$ than those recently reported for silica-encapsulated gold nanorods of similar size ${ }^{[27]}$ (blue dashed line in Figure 1e). This rise corresponds to an increase of $\Gamma_{\text {chem }}$ in the absence of silica encapsulation which provides nano-objects with a well-controlled interface ${ }^{[34]}$ (a similar $\Gamma_{\text {chem }}$ increase was measured after thiol functionalization of initially uncoated nanorods $\left.{ }^{[32]}\right) . \Gamma_{r}$ is negligible for the small nanorod sizes considered here, and $\Gamma_{0}$ is quasi-constant for SPR positions $\lambda_{R}>700 \mathrm{~nm}$ away from interband transition domain, as in the present study (Figure 1b-d). ${ }^{[27,35,36]}$ The weak dependence of $\Gamma$ on nanorod size (Figure 1e) thus essentially results from the quantum confinement term $\gamma_{s}$, which varies as $\gamma_{s}=A$ $v_{F} / L_{\text {eff }}$ (with $v_{F}$ Fermi velocity, $A=1.4$ and $L_{\text {eff }}$ the effective length related to the nano-object surface, $L_{\text {eff }}=(D L)^{1 / 2}$ for nanorod geometry). ${ }^{[27]}$ These broadening contributions are expected to be approximately the same for experiments on carbon substrates, which are performed by depositing nano-objects stemming from the same colloidal solutions and are also free from interband transition induced SPR broadening. The large increase of $\Gamma$ measured on carbon substrate can therefore be ascribed to its absorbing nature (with thus $\hbar \Gamma_{\text {sub }} \approx 135 \mathrm{meV}$ in Equation (1)), as confirmed by the complete analysis presented below.

Longitudinal extinction spectra measured on individual gold nanobipyramids with length and diameter in the $L=60-80 \mathrm{~nm}$ and $D=20-30 \mathrm{~nm}$ range are presented in Figure 2. Similarly to nanorods, analysis was performed by comparing single bipyramids with the same $\eta$ (Figure 2a) or $L_{\text {eff }}$ (Figure $2 \mathrm{~b}$ ) on carbon and silica, or by plotting $\Gamma\left(L_{e f f}\right)$ for all measurements (Figure $2 \mathrm{c}$ ). The SPR red shift measured for nanobipyramids of same aspect ratio is smaller than in the nanorod case (as illustrated by the $\Delta \lambda_{R} \approx 25 \mathrm{~nm}$ shift of Figure 2a). For nanobipyramids on silica, the average SPR width $(\hbar \Gamma=(135 \pm 20) \mathrm{meV})$ is the same as for nanorods on silica in spite of larger dimensions, due to a compensation of radiative damping (increasing with size) and 

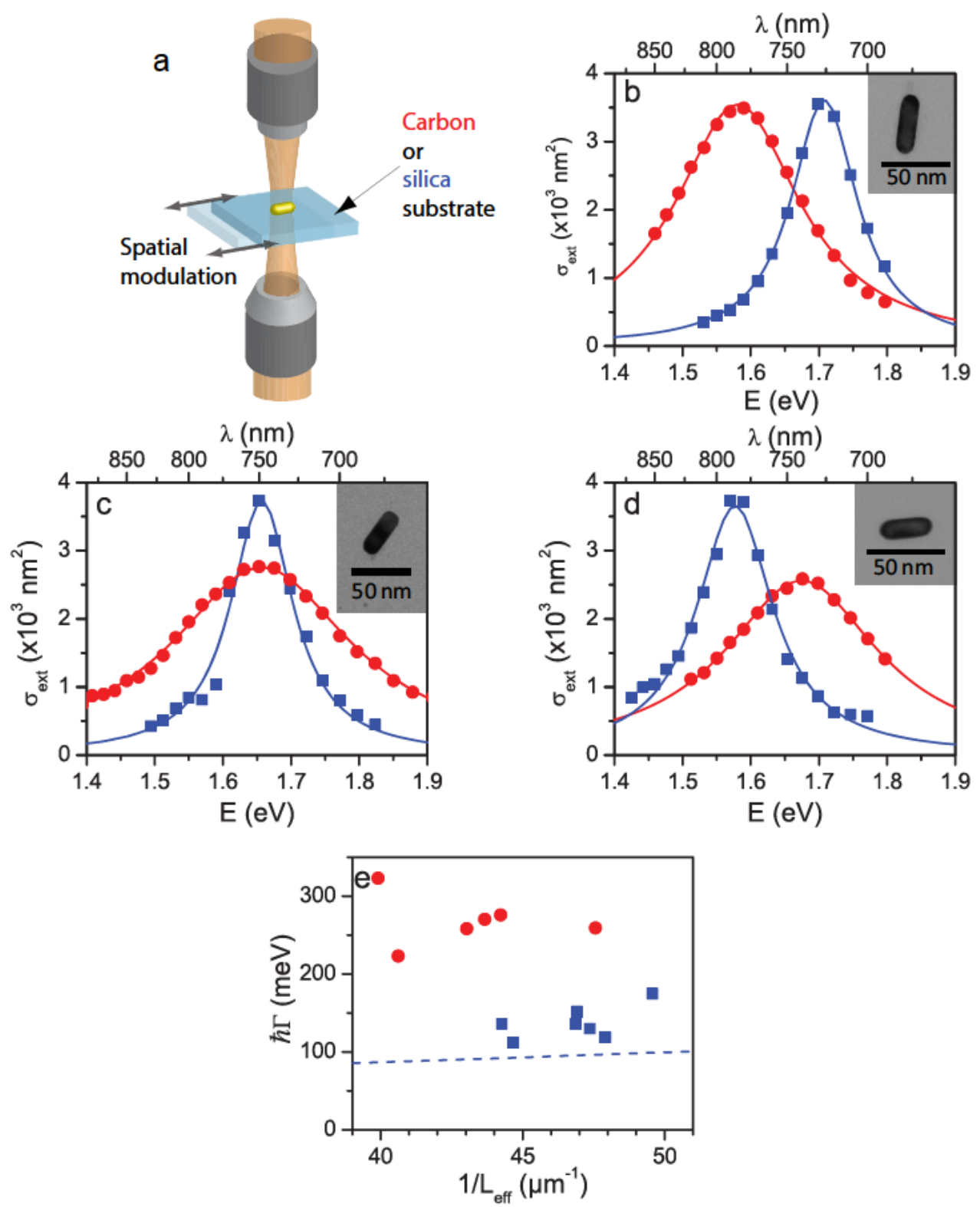

Figure 1. a) Principle of single particle spatial modulation spectroscopy, based on far-field illumination with a strongly focused laser beam, periodic modulation of nano-object position under the beam, and synchronous detection of the induced transmission change. b-d) Extinction spectra measured for single gold nanorods (of length $L$ and diameter $D$ ) on silica (blue squares) and carbon (red circles) substrates, with light polarization parallel to their long axes. Comparison is done at same nanorod aspect ratio $\eta=L / D=3$ (b), same SPR wavelength $\lambda_{R}=750 \mathrm{~nm}$ (c), and same effective length $L_{\text {eff }}=(L D)^{1 / 2}=23 \mathrm{~nm}$ (d). In each case, the TEM image in inset shows the morphology of the nanorod investigated on the carbon substrate (red spectrum), obtained after one-to-one correlation of the measured nanorod optical response with its electron microscopy image. Lorentzian fits (Equation (2)) of these spectra are shown by solid lines. e) Widths $\Gamma$ measured for different single nanorods on silica (blue squares) and carbon (red circles) substrates. The blue dashed line represents the size-dependent width previously measured for silica-encapsulated gold nanorods (with SPR far from interband transitions). ${ }^{[27]}$

quantum confinement effects (decreasing with size), leading to similar broadening contributions $\Gamma_{r}+\gamma_{s}$. However, the additional broadening effect due to carbon deposition is significantly less pronounced than for nanorods, with average widths of $\hbar \Gamma=(180 \pm 20) \mathrm{meV}$ for bipyramids on carbon (leading to $\left.\hbar \Gamma_{\text {sub }} \approx 45 \mathrm{meV}\right)$ as compared to $\hbar \Gamma=(270 \pm 30) \mathrm{meV}$ for nanorods on carbon $\left(\hbar \Gamma_{\text {sub }} \approx 135 \mathrm{meV}\right)$. This difference highlights the need for complete modeling of the optical response of elongated nano-objects deposited on an absorbing substrate, taking into account the details of their 3D geometry and orientation.

\subsection{Modeling of Extinction Spectra}

As discussed in previous theoretical works, the extinction of a light beam by a nano-object placed in an absorbing environment is the sum of three contributions: absorption within the 

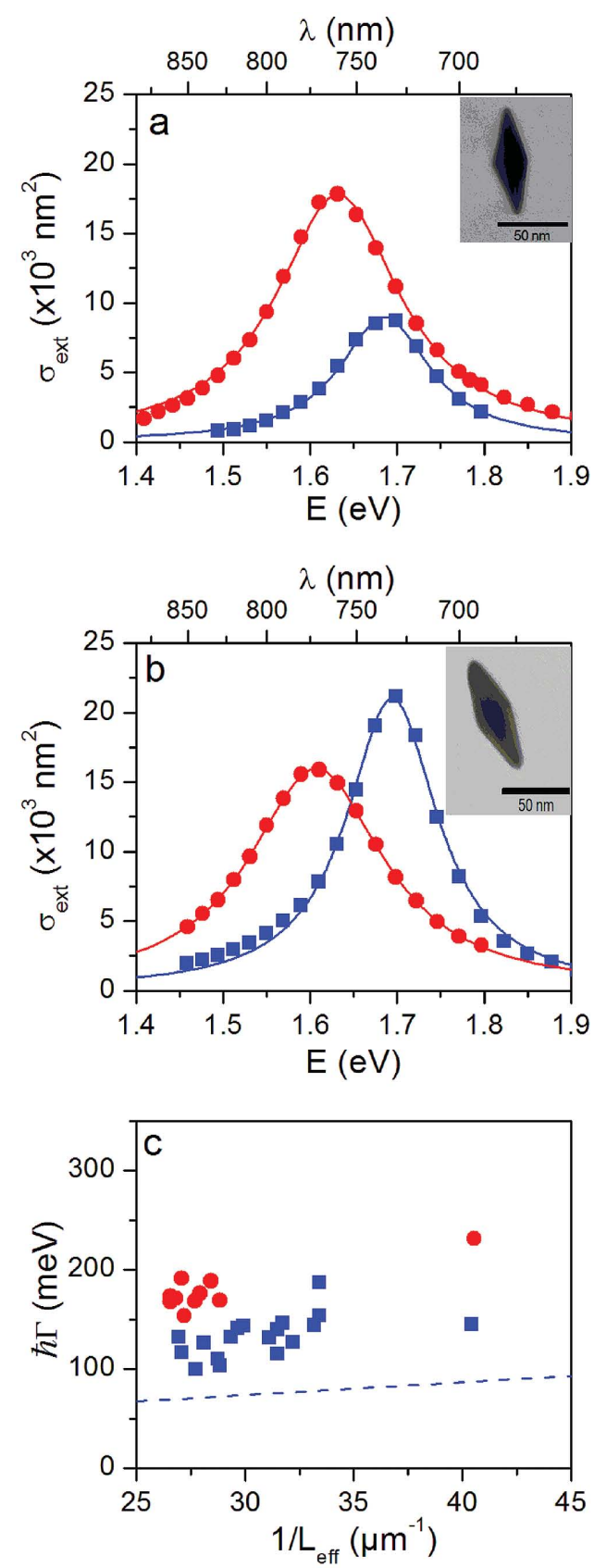

Figure 2. a-b) Longitudinal extinction spectra measured for single gold nanobipyramids on silica (blue squares) and carbon (red circles) substrates for same aspect ratio $\eta=2.7$ (a) and same $L_{\text {eff }}=36 \mathrm{~nm}$ (b). Lorentzian fits (Equation (2)) of these spectra are shown by solid lines. TEM images of the nanobipyramids on carbon are shown in inset. c) SPR widths measured for individual nanobipyramids on silica (blue squares) and carbon (red circles) substrates. For comparison, the blue dashed line represents the size-dependent width previously measured for silica-encapsulated gold nanorods ${ }^{[27]}$ (as in Figure 1).

nanoparticle, far-field scattering, and enhanced environment absorption at the vicinity of the nano-object as a consequence of local field effects. ${ }^{[37,38]}$ The extinction cross-section $\sigma_{\text {ext }}$ of a nano-object in an absorbing medium is proportional to the difference between the local power losses due to the nanoparticle (absorption and scattering) and the absorption ones in the same nano-object volume filled with the environment medium. ${ }^{[37,38]}$ In order to qualitatively understand how the characteristics of an absorbing environment affect the longitudinal SPR of an elongated nano-object, it is helpful to first consider the simple case of small nanoellipsoids in a homogeneous infinite medium with dielectric constant $\varepsilon^{m}=\tilde{n}_{m}^{2}$. In the quasistatic regime (i.e., for nano-object size much smaller than optical wavelength), when the SPR is located away from the interband transition domain, the frequency dependence of $\sigma_{\text {ext }}$ is well described by a Lorentzian lineshape (see Supplementary Information) characterized by three independent parameters (integrated area $\Xi$, central frequency $\Omega_{R}=2 \pi \mathrm{c} / \lambda_{R}$ and spectral width $\Gamma$ ):

$$
\sigma_{\text {ext }}(\omega)=\Xi \frac{\Gamma /(2 \pi)}{\left(\omega-\Omega_{R}\right)^{2}+(\Gamma / 2)^{2}}
$$

The integrated area $\Xi$ is proportional to the nanoparticle volume, and $\Omega_{R}$ and $\Gamma$ are given by:

$$
\left\{\begin{array}{c}
\Omega_{R}=\frac{\omega_{p}}{\sqrt{\varepsilon_{1}^{i b}+\varepsilon_{1}^{m}\left(\frac{1}{L_{i}}-1\right)}} \\
\Gamma=\frac{\Omega_{R}^{3}}{\omega_{p}^{2}}\left[\varepsilon_{2}+\left(\frac{1}{L_{i}}-1\right) \varepsilon_{2}^{m}\right]
\end{array}\right.
$$

with $\omega_{p}$ plasma frequency and $L_{i}$ geometrical factors depending on ellipsoid aspect ratio and light polarization. The SPR spectral position and width are therefore directly affected by the real $\left(\varepsilon_{1}^{m}\right)$ and imaginary $\left(\varepsilon_{2}^{m}\right)$ parts of the absorbing environment dielectric constant $\varepsilon^{m}$, respectively.

The optical response of carbon, i.e., its complex refractive index $n^{C}=n_{1}{ }^{C}+\mathrm{i} n_{2}{ }^{C}$, is therefore a crucial input for a quantitative analysis of the measured extinction spectra. Previous optical measurements on thin carbon films yielded $n_{1}{ }^{C}=2.3 \pm 0.3$ (with a weak dispersion in the visible range). ${ }^{[39-41]}$ Much more scattered $n_{2}{ }^{C}$ values, in the $0-1$ range, were reported, possibly due to the different preparation methods and structures (amorphous, diamond-like, nanocrystalline...) of the investigated carbon films. ${ }^{[39-41]}$ In this work, instead of using tabulated values, we directly extracted this parameter by measuring light attenuation through the carbon membranes, yielding $n_{2}{ }^{C} \approx 0.5$ (see Figure S1 and text of the Supplementary Information).

For a more detailed modeling, FEM simulations taking into account actual nano-object shapes and their inhomogeneous environment, as well as finite-size effects (i.e., radiative damping, retardation effects and multipolar contributions) were performed (Figure 3 and Figure 4). As in previous reports, nanorods were described as cylinders capped by hemispherical ends (Figure 3a), while nanobipyramids were represented as truncated bicones with hemispherical ends ${ }^{[15]}$ (Figure 4a). Typical experimental sizes were used in simulations: $L=42 \mathrm{~nm}$ and $D=14 \mathrm{~nm}$ for the length and diameter of nanorods, and $L=78 \mathrm{~nm}, D=29 \mathrm{~nm}$ and $r=4 \mathrm{~nm}$ tip radius for bipyramids. Simulations were performed using Johnson and Christy dielectric functions for bulk gold, ${ }^{[42]}$ without including surface and broadening contributions $\left(\Gamma_{\text {chem }}=\gamma_{s}=0\right.$ in Equation (1)). A 

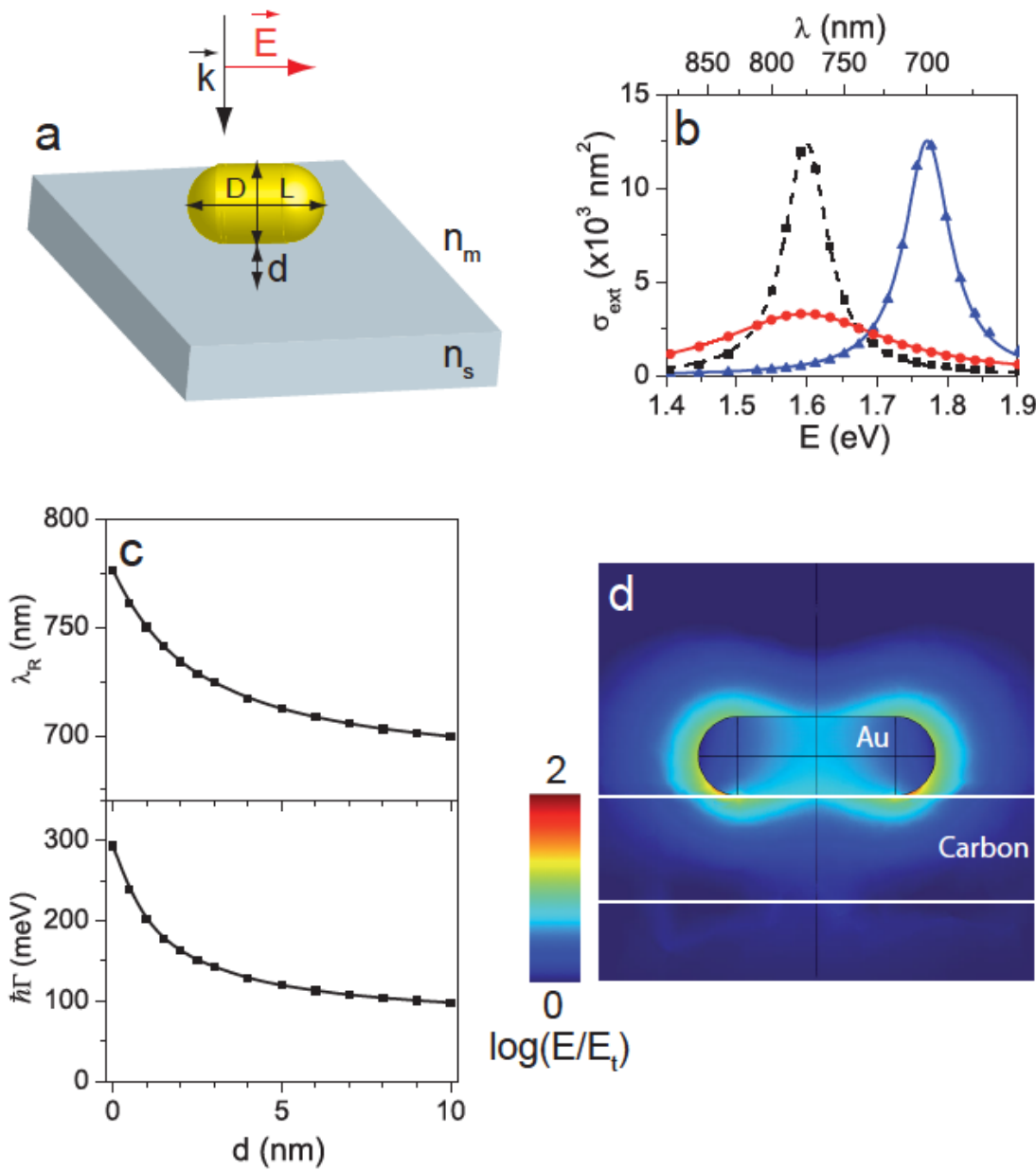

Figure 3. a) Geometry used for optical FEM simulations: a horizontally oriented gold nanorod (length $L$, diameter $D$ ) in a transparent medium (refractive index $n_{m}$ ) standing at distance $d$ from a membrane (refractive index $n_{5}$ ) and illuminated by a vertically propagating plane wave polarized along the long axis of the rod. b) FEM-computed longitudinal extinction spectra for a $L=42 \mathrm{~nm}, D=14 \mathrm{~nm}$ nanorod in a $n_{m}=1.3$ medium and in direct contact $(d=0)$ with $n_{s}=1.45$ (blue triangles), $n_{s}=2.3$ (black squares) and $n_{s}=2.3+0.5 \mathrm{i}$ (red circles) $20 \mathrm{~nm}$ thick substrates. The lines are Lorentzian fits (Equation (2)). c) $d$-dependence of SPR position $\lambda_{R}$ and width $\Gamma$ for carbon substrate. d) Computed electric field distributions at SPR for carbon substrate $(d=0)$.

$20 \mathrm{~nm}$ thick membrane with $n_{\mathrm{s}}=2.3+0.5$ i for the carbon substrate and $n_{s}=1.45$ for the silica one was considered, together with a local environment refractive index $n_{m}=1.3$ (which takes into account, consistently with experiments, the presence of surfactants and/or water around the nano-objects ${ }^{[34]}$ ). Extinction cross-sections were calculated from the computed electromagnetic field distribution, defining $\sigma_{e x t}=P_{e x t} / I_{t}=\left(P_{a b s}^{A u}+P_{\text {scat }}\right) / I_{t}$, with $I_{t}$ the intensity of the plane wave transmitted through the substrate and $P_{\text {ext }}$ the rate of incident beam energy removal induced by the particle. ${ }^{[37,38]}$ The latter is decomposed as the sum of the power absorbed in the nano-object $P_{a b s}^{A u}$ (computed by volumic integration of Ohmic losses) and $P_{\text {scat }}$, which lumps far-field scattering and modification of substrate absorption.

Quasi-Lorentzian $\sigma_{e x t}$ spectra in the energy domain were obtained, and fitted using Equation (2) (Figure 3b and 4b). Comparison of calculations performed with substrate refractive indexes of silica ( $n_{s}=1.45$, blue triangles), carbon $\left(n_{s}=2.3+0.5 \mathrm{i}\right.$, red circles) and with $n_{\mathrm{s}}=2.3$ (black squares) shows that the real and imaginary parts of $n_{s}$ predominantly impact SPR spectral position and width, respectively, confirming the simplified analytical calculations above (Equation (3)). As expected, the impact of environment on $\lambda_{R}$ and $\Gamma$ strongly depends on the distance $d$ separating the nano-object and the membrane (Figure 3c and 4c). For nanorods in contact with the membrane, substrate-induced SPR red shifts up to about $100 \mathrm{~nm}$ and broadening up to $200 \mathrm{meV}$ are expected (Figure 3c). The situation is more complex for bipyramids, for which FEM simulations reveal the strong impact of the deposition angle $\alpha$ (Figure $4 \mathrm{a}$ ). In this case, much larger substrate effects are predicted for maximally tilted $\left(\alpha=\alpha_{\max }=\operatorname{atan}((D-2 r) /\right.$ (L-2r))) bipyramids than for horizontally orientated $(\alpha=0)$ ones (Figure $4 \mathrm{~b}-\mathrm{d})$. In the tilted configuration, the lateral surface of the bipyramid is in contact with the substrate, and red shifts and broadening are close to those for nanorods.

These effects, weakly dependent on membrane thickness in the $10-40 \mathrm{~nm}$ range, can be qualitatively understood from the spatial distribution of electromagnetic fields at SPR (Figure 3d and Figure 4e-f), considering that the longitudinal SPR of an elongated nanoobject is mostly sensitive to the local properties of the external medium at the vicinity of its ends, where large electromagnetic fields are generated. In the nanorod case, these regions overlap with the carbon membrane for small nano-object-substrate distance $d$ (Figure 3d). This induces a large SPR red shift (as the real part of carbon is much larger than the environment one) and broadening, associated to strong local enhancement of the membrane absorption[20] (Figure 3c). Tip electromagnetic fields are more intense and localized for nanobipyramids, due to the smaller radius of their ends as compared to nanorods. Therefore, significant penetration of large fields in the membrane is achieved at the lowest end only for large inclination $\left(\alpha \approx \alpha_{\max }\right)$ and small $d$. For other values of $\alpha$ and $d$, and in particular for horizontal orientation, this situation is not expected to occur, leading to moderate SPR red shift and broadening.

For gold nanorods, the experimental substrate-induced broadening $\left(\hbar \Gamma_{\text {sub }} \approx 135 \mathrm{meV}\right)$ is obtained in simulations for $d=(1.5 \pm 1) \mathrm{nm}$, which is close to the expected thickness of the CTAB surfactant layer around the gold nano-objects (about $\left.2 \mathrm{~nm}^{[43,44]}\right)$. The weak broadening measured for bipyramids is compatible with that predicted for a bipyramid at the same distance from the carbon substrate with a horizontal configuration $(\alpha=0)$. This effect could also be achieved for a maximally tilted orientation $\left(\alpha=\alpha_{\max }\right)$, but only for a large distance between 

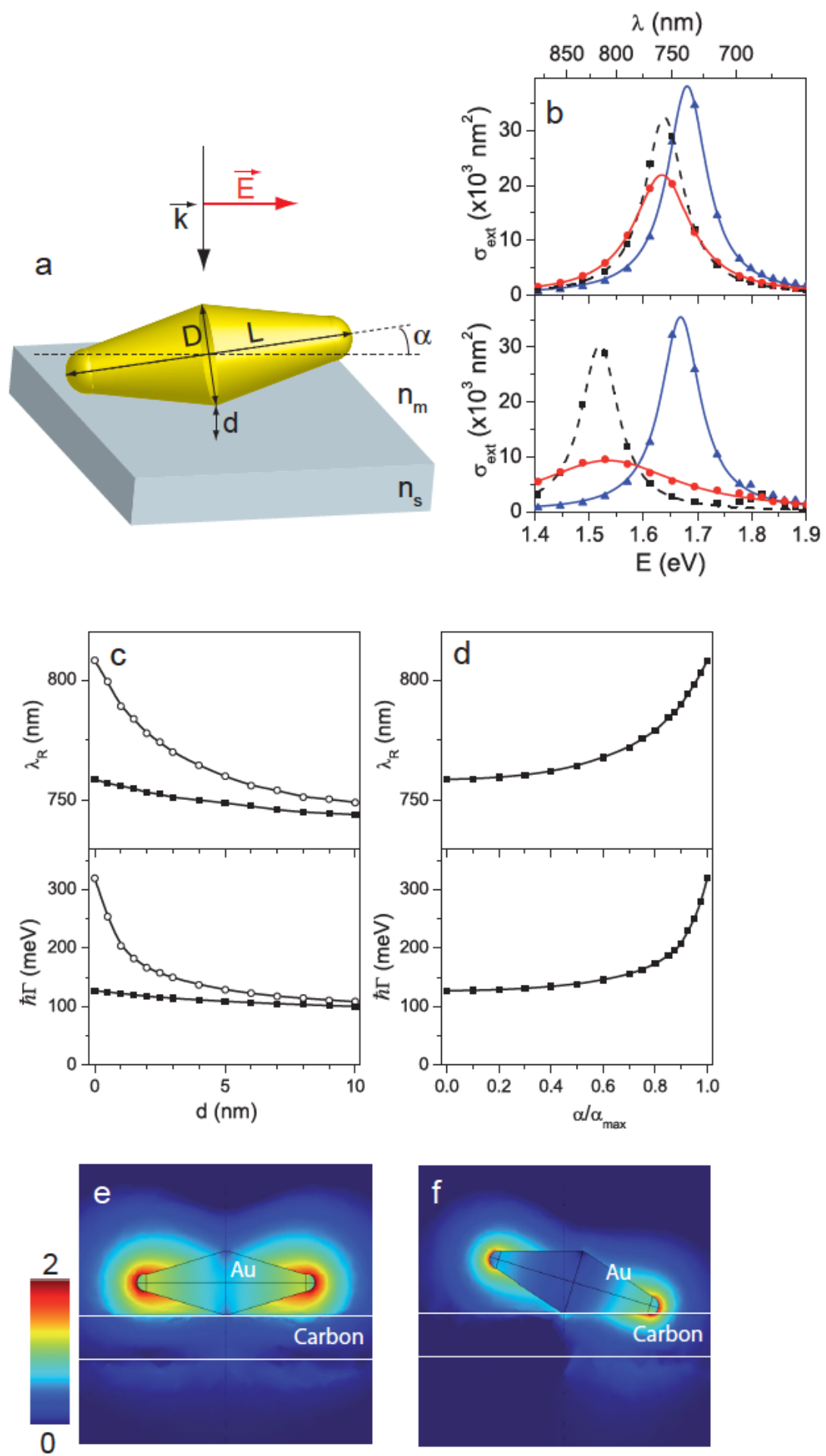

$\log \left(E / E_{t}\right)$

Figure 4. a) Geometry used for optical FEM simulations of a bipyramid (orientation angle $\alpha$ and other parameters as in Figure 3). b) FEM-computed longitudinal extinction spectra for $L=78 \mathrm{~nm}$, $D=29 \mathrm{~nm}, n_{m}=1.3$ and direct contact $(d=0)$ with $n_{s}=1.45$ (blue triangles), $n_{s}=2.3$ (black squares) and $n_{s}=2.3+0.5 \mathrm{i}$ (red circles) $20 \mathrm{~nm}$ thick substrates for horizontal ( $\alpha=0$, top) and maximally tilted ( $\alpha=\alpha_{\max }$, bottom) orientations. The lines are Lorentzian fits (Equation (2)). c) $d$-dependence of $\lambda_{R}$ and $\Gamma$ on a carbon membrane for horizontal (squares) and maximally tilted (open circles) orientations. d) $\alpha$-dependence of these parameters for $d=0$. e) Computed electric field distribution at SPR for a carbon membrane with $d=0$ and $\alpha=0$. f) Same for $\alpha=\alpha_{\max }$. the nano-object and the substrate $(d>7 \mathrm{~nm}$, Figure 4c), which appears unlikely in view of the actual surfactant layer thickness around the nano-objects.

\subsection{Electron Tomography}

In order to extract information on the precise $3 \mathrm{D}$ orientation of the nano-objects with respect to the carbon substrate, and therefore yield both $d$ and $\alpha$ parameters, we performed 3D electron tomography, a technique based on the acquisition of a series of tilted 2D TEM images combined with reconstruction algorithms using Radon theory. ${ }^{[45,46]}$ Precise localization of the substrate plane on the resulting images was achieved through deposition of small markers (5 nm gold nanoparticles), which can be detected with high contrast in electron microscopy (Figure $\mathbf{5 a}-\mathrm{c}$ ). For all the bipyramids investigated using electron tomography, images indicate a horizontal orientation $(\alpha \approx 0)$ with a $d=(2 \pm 0.5) \mathrm{nm}$ nanobipyramid-substrate separation (Figure $5 c$ ), of the order of the thickness of surfactant coating layer.

Note that, on glass substrate, an inclined bipyramid configuration was observed using AFM height profile measurements in other experiments. ${ }^{[47]}$ This difference in orientation can possibly result from differences in synthesis and surfactant coating, but also from different interactions of the nano-objects with the substrate. A direct electron tomography measurement of $3 \mathrm{D}$ orientation of bipyramids on silica substrates would be very interesting, but remains challenging (e.g. due to charging of the silica membrane under prolonged electron irradiation). However, the precise $3 \mathrm{D}$ orientation of nano-objects on transparent substrates only plays a minor role for the specific broadening effects investigated here.

The direct comparison between a single bipyramid extinction spectrum and FEMcomputed spectra on carbon, assuming the horizontal configuration determined using electron tomography, is presented in Figure 5d. Simulations were performed using TEM sizes and taking into account all contributions discussed in Equation (1) (including radiative, surface and substrate effects and using $\Gamma_{0}+\Gamma_{\text {chem }}+\gamma_{\mathrm{s}} \approx 100 \mathrm{meV}$, as deduced from previous experiments on gold nano-objects ${ }^{[15,27,34]}$ ) and $n_{m}$ adjusted to precisely reproduce the SPR position (with $n_{m} \approx 1.3$ for the TEM-determined horizontal configuration, this value accounting 


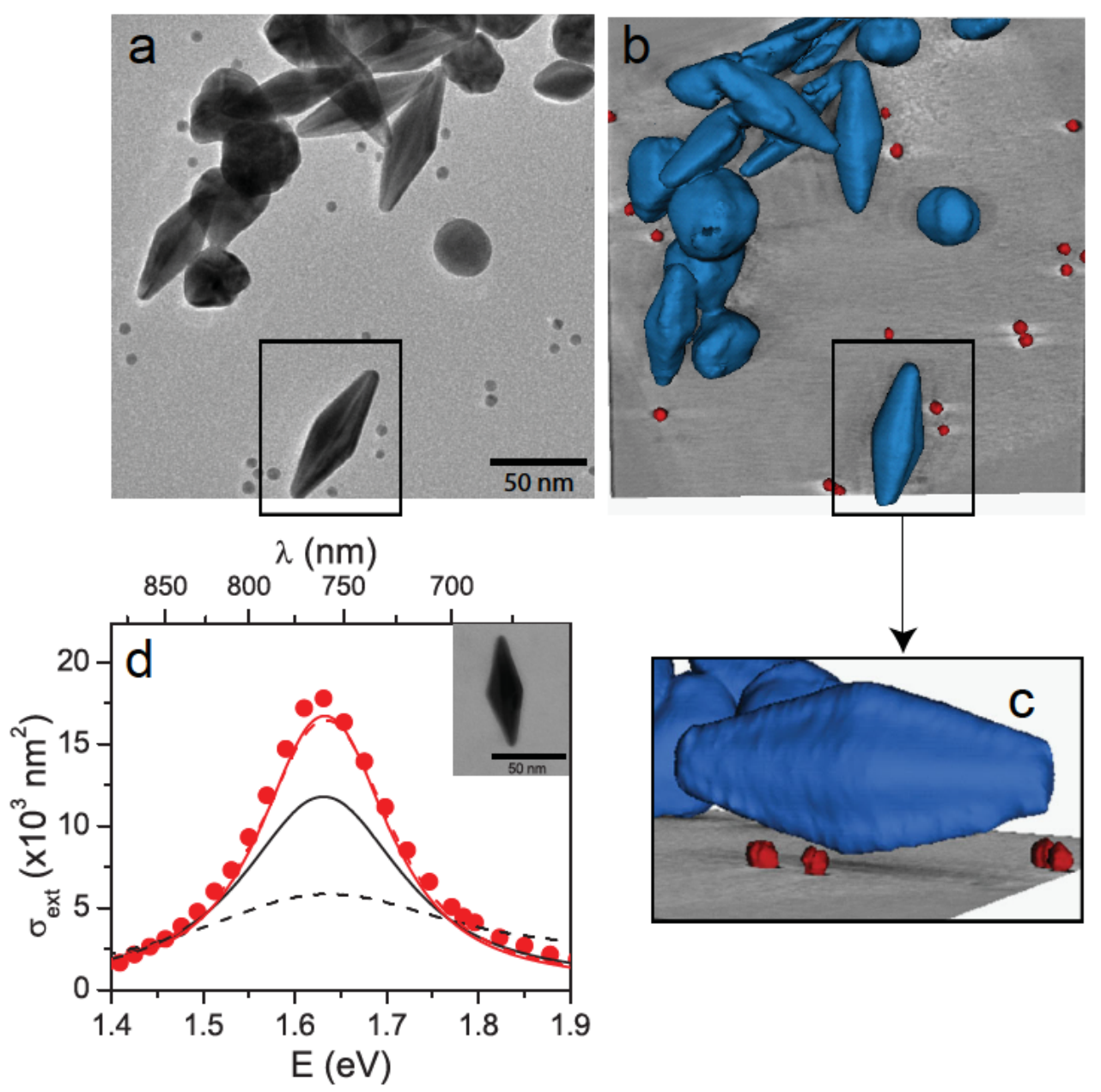

Figure 5. a-c) Electron tomography analysis of the orientation of gold bipyramids deposited on carbon substrates, using small gold nanoparticles as markers. a) TEM image of the analyzed zone at $0^{\circ}$ tilt. b) 3D representation of the same region, obtained by applying a data segmentation procedure to the reconstructed volume (gold nano-objects from the colloidal solution are in blue and markers in red). c) Lateral view of the 3D reconstructed bipyramid and neighbor markers, demonstrating its horizontal orientation $(\alpha \approx 0)$ with $d \approx 2 \mathrm{~nm}$ separation from the substrate. d) SMS extinction spectrum (red circles) of a $L=78 \mathrm{~nm}, D=29 \mathrm{~nm}$ and $r=4 \mathrm{~nm}$ gold nanobipyramid (dimensions deduced from the TEM image of this nanobipyramid, shown in inset) and corresponding FEM calculations with same sizes using the horizontal 3D configuration ( $\alpha=0$ and $d=2 \mathrm{~nm}$, red line). Also shown are calculations with $\alpha=0$ and $d=0 \mathrm{~nm}$ (dashed red line, similar to previous one), and for a tilted bipyramid: $\alpha=\alpha_{\max }$ and d =2 nm (solid black line) nd $\alpha=\alpha_{\max }$ and $\mathrm{d}=0 \mathrm{~nm}$ (dashed black line).

in particular for the presence of the CTAB layer surrounding the bipyramid). Comparison shows that the spectrum computed for the horizontal orientation with $d=2 \mathrm{~nm}$ (solid red line) is in good quantitative agreement with the experimental one, displaying similar quasi-Lorentzian shape, area and width. Interestingly, this agreement is obtained without including in the modeling the charge transfer effects invoked in the analysis of the scattering spectra of gold nanorods on graphene, ${ }^{[21]}$ suggesting that these effects play a negligible role in our study. It is maintained if a smaller $d$ value is used in the calculations (see dashed red line corresponding to $d=0 \mathrm{~nm}$, almost overlapping the previous one), which results from the weak sensitivity on $d$ of the SPR properties of a horizontal nanobipyramid due to strong field localization at its tips (Figure 4c,e). Conversely, the expected spectra for a maximally tilted bipyramid (black lines) are broader than the experimental one for both distances ( $d=2 \mathrm{~nm}$, solid line and $d=0 \mathrm{~nm}$, dashed line).

\subsection{Extension to Au@Ag Nano-objects}

To confirm the effect of 3D orientation and contact with an absorbing substrate, additional optical and electron tomography measurements were performed on silver-encapsulated gold bipyramids, whose shape can be controlled from that of a bipyramid toward that of a rod by varying the amount of silver in the shell[43] (see Figure 6 and Experimental Section for details on synthesis). Two extinction spectra of single Au@Ag nanoobjects deposited on carbon with similar sizes but different shell thicknesses (corresponding to $30 \%$ and $60 \%$ silver volume fraction) are shown in Figure 6a. The electron tomography image of the one presenting the thickest shell is shown in Figure 6b. The longitudinal SPR position $\lambda_{R}$ is blue shifted upon silver coating, due to modification of the nano-object metal dielectric function (see simulations below) and in agreement with previous experimental reports on Ag-coated Au nanorods. ${ }^{[48,49]}$ The 

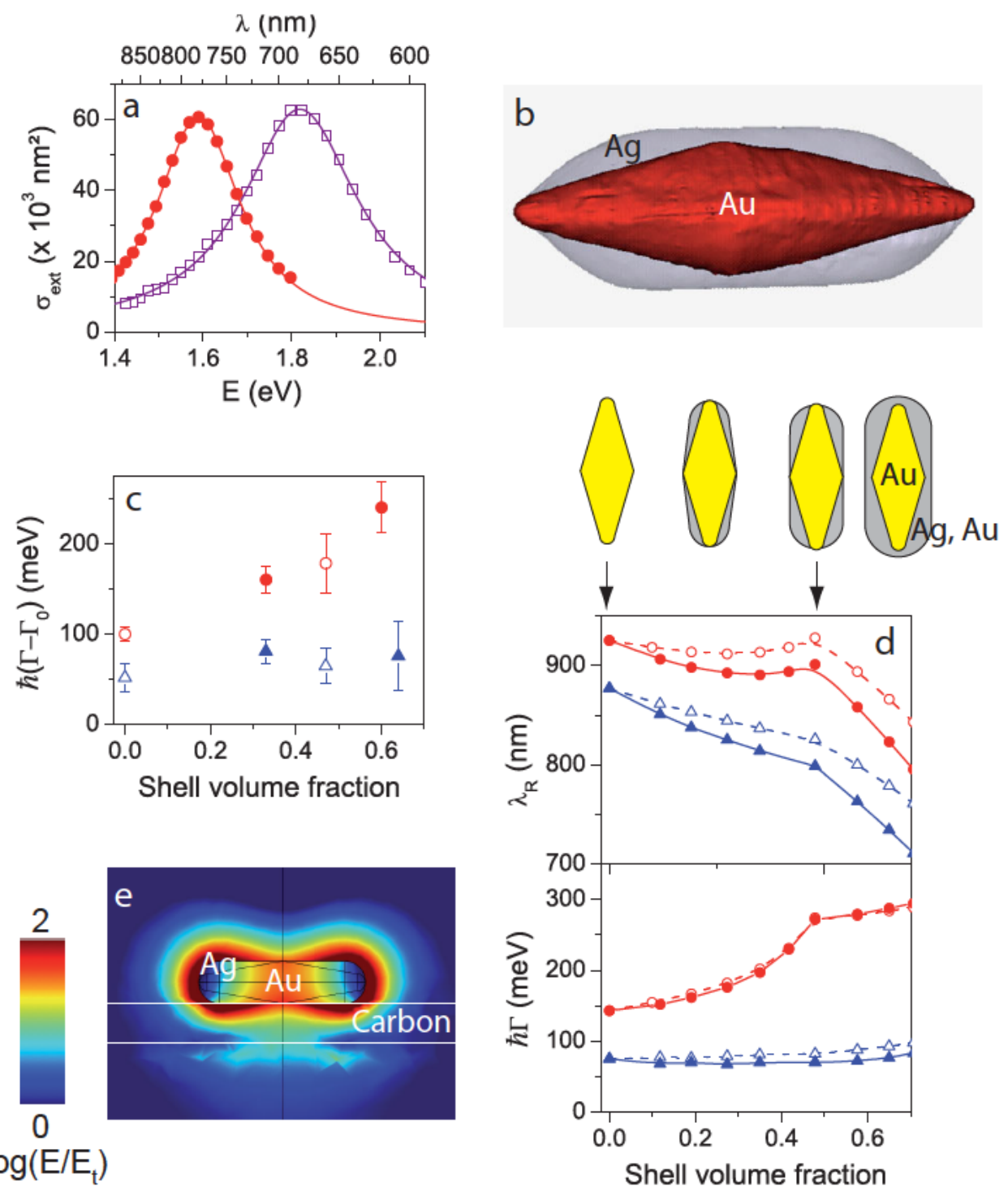

Figure 6. a) Extinction spectra of individual Au@Ag nano-objects with thin ( $\approx 30 \%$ volume fraction, red circles) and thick $(\approx 60 \%$ volume fraction, purple open squares) silver shell on carbon substrates. The total length and diameter ( $\approx 135$ and $35 \mathrm{~nm}$, respectively) of the two nano-objects measured by TEM differ by less than $5 \%$. Solid lines are Lorentzian fits (Equation (2)). b) Electron tomography image of the Au@Ag nano-object with the thick silver shell (purple spectrum in a). c) Mean SPR width measured for different single gold and silver-encapsulated nano-objects on carbon (red circles) and silica (blue triangles). Empty symbols correspond to pure Au nano-objects (i.e., nanobipyramids and nanorods, the latter can be considered here as gold bipyramids encapsulated with gold shell) and solid ones to silver-encapsulated gold nanobipyramids. The bulk-like Landau damping contribution $\Gamma_{0}$ was subtracted to suppress interband broadening occurring when $\lambda_{R}<700 \mathrm{~nm}$. d) FEM-computed SPR position (top) and width (bottom) of encapsulated gold bipyramids ( $L=80 \mathrm{~nm}, D=20 \mathrm{~nm}, r=4 \mathrm{~nm}$ ) on carbon (red circles) and silica (blue triangles) membranes as a function of shell volume fraction, for silver (solid symbols and lines) or gold (empty symbols and dashed lines) shells. e) Computed electric field distribution at SPR for Au@Ag nanobipyramid in contact with carbon with a cylindrical silver shell with same $L$ and $D$ as the Au core (0.47 silver volume fraction), corresponding to a rod-like external shape.

presence of the silver shell induces a large broadening of $\sigma_{e x t}$ spectra, as compared to pure gold ones, increasing with silver volume fraction (see Figure 6c, circles). For the thickest silver coating (Figure 6a, purple open squares), $\Gamma$ is on average two times larger $((320 \pm 40) \mathrm{meV})$ than for pure gold bipyramids $(180 \mathrm{meV})$, a value even exceeding the one measured for gold nanorods $(270 \mathrm{meV})$.

The large broadening observed on carbon membranes when increasing silver coating may a priori be associated to either a composition (i.e. addition of another metal) or a shape effect, as Au@Ag nano-objects evolve from a nanobipyramid to a nanorod shape with silver encapsulation (Figure 6b). In order to disentangle these two mechanisms, similar experiments were performed with the same nano-objects deposited on silica. Apart from an expected interband broadening for nanopyramids with the thickest shell (whose spectral position $\lambda_{R} \approx 620 \mathrm{~nm}$ is close to interband transitions, leading to a wavelength-dependent increase of the bulk-like contribution 
$\left.\Delta \Gamma_{0} \approx 60 \mathrm{meV}^{[27,35,36]}\right)$, no significant modification of the SPR width upon silver coating was observed on silica (Figure 6c, where $\Gamma_{0}$ has been subtracted to $\Gamma$ for direct comparisons). The absence of metal coating-induced effects on transparent substrate suggests that the main mechanism at the origin of broadening is an increased interaction with the absorbing substrate due to nano-object shape evolution.

To confirm this hypothesis and estimate the impact of these phenomena on $\lambda_{R}$ and $\Gamma$, we performed FEM simulations on horizontally orientated gold bipyramids (morphology described by $L=80 \mathrm{~nm}, D=20 \mathrm{~nm}$ and $r=4 \mathrm{~nm}$, a high $L / D$ ratio being chosen to avoid interband SPR broadening), encapsulated with silver or gold shells (leading in the latter situation to pure gold nano-objects, thus permitting a selective study of shape effects). Nano-objects with small shells were modeled as bipyramids with the same $L$ and $D$ values as the core one but larger tip radius of curvature $r$, yielding a nanorod shape for $r=D / 2$ and shell volume fraction $\approx 47 \%$ (Figure $6 \mathrm{~d}$ ). Larger shells were described by increasing $L$ and $D$ of the same amount (thus modifying the aspect ratio of the nano-object). Comparison between computations performed for Au@Ag and Au@Au nano-objects indicates that metal composition affects $\lambda_{R}$ (silver coating, solid symbols of Figure $6 \mathrm{~d}$, inducing as expected ${ }^{[48,49]}$ a supplementary blue shift of the longitudinal SPR as compared to gold coating, empty symbols of Figure 6d) but does not significantly modify $\Gamma$. Conversely, increasing the shell (gold or silver) volume fraction results in a large increase of $\Gamma$ for nanoobjects deposited on carbon (circles of Figure 6d, bottom), as compared to silica. This confirms that the large experimental increase of $\Gamma$ on carbon membranes upon silver encapsulation can be ascribed to the evolution of nano-object shape from a horizontal bipyramid to a nanorod one. The latter shape results in larger SPR width as discussed previously (Figure 1 and 2), related to the larger penetration in the absorbing membrane of the intense electric field regions (Figure 6e), similarly to the nanorod case (Figure 3d). While theoretical trends are in qualitative agreement with experiments, a more quantitative comparison is however difficult due to uncertainties on the tabulated dielectric functions of silver ${ }^{[50]}$ and on possible additional chemical damping contributions in the presence of Au-Ag interfaces.

\section{Conclusion}

In conclusion, comparing the optical responses of individual nanorods and bipyramids deposited on a dielectric and absorbing substrate, we have quantitatively investigated the impact of the substrate on the SPR of a nano-object and highlighted the critical dependence on its shape, 3D orientation and distance from it. This is a consequence of modification of the local electromagnetic field around the nano-object, in the vicinity of its tips in the case of an elongated one, which reflects in the observed SPR characteristics. In particular, damping of the SPR is larger for a rod than for a bipyramid deposited on an absorbing substrate due to weaker localization of local field enhancement in a rod, leading to larger enhancement of the local field in the substrate and thus of its absorption. These results are in excellent agreement with numerical simulations using the nano-object morphology and position relative to the substrate determined by electron microscopy. The key geometrical parameters are the curvature of the object tips, which rules the amplitude and localization of the electromagnetic field, and their distance to the substrate, parameters that can be determined using three dimensional imaging of both the nanoobject and the substrate with electron tomography. Sensitivity of the SPR to these parameters stresses the importance of detailed characterization of the investigated nano-object and local, i.e. nanometric scale, environment when interpreting its optical response. These results could also facilitate the conception of efficient (i.e., avoiding significant performance degradation by SPR broadening) plasmonic devices in absorbing environments, especially for nano-objects prepared on carbon substrate, common in many nano-system fabrication procedures.

\section{Experimental Section}

Chemicals: Tetrachloroauric acid $\left(\mathrm{HAuCl}_{4} \times 3 \mathrm{H}_{2} \mathrm{O}\right)$, tetraethylorthosilicate (TEOS), absolute ethanol, $\mathrm{NH}_{4} \mathrm{OH}$ (29\%), sodium borohydride $\left(\mathrm{NaBH}_{4}\right)$, ascorbic acid (A.A.), silver nitrate $\left(\mathrm{AgNO}_{3}\right)$, O-[2(3-mercaptopropionylamino)ethyl]-O'-methyl-poly (ethylene glycol) (PEG$\mathrm{SH}, \mathrm{Mw} 5000), \mathrm{HCl}(37 \%)$, and trisodium citrate were purchased from Sigma-Aldrich. All chemicals were used as received. Milli-Q grade water was used as solvent.

Synthesis of Gold Bipyramids: Gold bipyramids were prepared by the seeded growth method previously reported in literature. ${ }^{[51]}$ Gold seeds were prepared as follows: $300 \mu \mathrm{L}$ of $\mathrm{NaBH}_{4}(10 \mathrm{mM})$ were quickly added to a solution of $0.125 \mathrm{mM}$ of $\mathrm{HAuCl}_{4}$ and $0.25 \mathrm{mM}$ of $\mathrm{Na}_{3} \mathrm{Cit}$ under vigorous stirring. Seeds $(300 \mu \mathrm{L})$ were added to the growth solution $(10 \mathrm{~mL})$ typically formed by CTAB $0.1 \mathrm{M}, \mathrm{HAuCl}_{4}(0.5 \mathrm{mM}), \mathrm{AgNO}_{3}(0.1 \mathrm{mM})$, $\mathrm{HCl}(20 \mathrm{mM})$ and A.A. $(0.8 \mathrm{mM})$ as mild reducing agent. The reaction was kept at $30{ }^{\circ} \mathrm{C}$ during 2 hours. Bipyramids were washed by centrifugation $\left(8000 \mathrm{rpm}, 20^{\prime}\right)$ two times, the supernatant was discarded and the precipitate was redispersed in water in order to have a CTAB concentration of $1 \mathrm{mM}$. This approach allows production of bipyramids with a $\sim 80 \%$ yield, with spherical nanoparticles as by-products. The as-prepared elongated $\mathrm{Au}$ nano-objects are capped with a dense bilayer of amphiphilic molecules (CTAB), with a thickness of about $2 \mathrm{~nm}$ which becomes increasingly sparse at the tips, as shown in a previous work. ${ }^{[52]}$

Encapsulation of Gold Bipyramids with Silver: The growth of $\mathrm{Ag}$ on gold bipyramids was carried out using a growth solution prepared with $0.1 \mathrm{mM}$ of gold bipyramids, $0.05 \mathrm{M}$ of CTAB, ascorbic acid in a relation of [AA]/ $\left[\mathrm{Ag}^{+}\right]=20$ and silver nitrate, whose quantity was varied in order to produce different sizes of the silver shell around the gold bipyramids. To start the silver reduction, $0.01 \mathrm{M} \mathrm{NaOH}$ was added until basic $\mathrm{pH}$ $(\mathrm{pH} \approx 9)$, and the reaction was kept in a thermostatic bath during $2 \mathrm{~h}$. The obtained nanoparticles were washed by centrifugation $(8000 \mathrm{rpm}$, 20 ) two times, the supernatant was discarded and the precipitate was redispersed in water, yielding a CTAB concentration of $1 \mathrm{mM}$. To avoid the oxidation of silver, a PEG coating was performed adding, under soft stirring, a PEG-SH solution $(1 \mathrm{mg} / \mathrm{mL})$ to the washed Au@ $\mathrm{Ag}$ bipyramids. The reaction was kept overnight. To remove the excess of polymer, further centrifugation (7000 rpm, 20') was performed, the supernatant was discarded and the pellet was redispersed in ethanol. Following this experimental procedure, the silver preferentially deposits onto the lateral facets of the gold elongated nano-objects. ${ }^{[45]}$ This phenomenon is assigned to the strong interaction between the silver precursor and the counter-ion of the surface-adsorbed amphiphilic surfactant (bromide ion from CTAB). The non-uniform CTAB distribution along the gold surface favors the preferential deposition of silver on the lateral facets and not at the tips. Consequently, the shape evolves from a bipyramid to a nanorod for thick silver coating. 
Spatial Modulation Spectroscopy: Colloidal solutions were spin-coated on TEM grids with $50 \times 50 \mu \mathrm{m}^{2}$ windows covered by either $a \approx 40 \mathrm{~nm}$ thick silica or $\approx 20 \mathrm{~nm}$ thick carbon membrane, deposition parameters

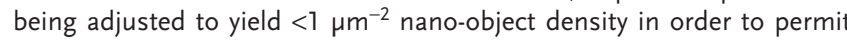
their optical separation. Low and higher magnification 2D TEM images of the samples were taken before and after optical measurements, respectively, in order to identify relevant, well-isolated individual nanoobjects without altering their optical response and to characterize their morphology. Their extinction cross-sections were quantitatively measured using spatial modulation spectroscopy (SMS). This technique is based on modulation at frequency $f$ of the position of a nano-object in the focal spot of a tightly focused light beam. Modulation of the transmitted light power is observed when a nano-object is present on the path of the illuminating beam, with an amplitude proportional to its absolute extinction cross-section $\sigma_{\text {ext }}(\lambda)$ at the light wavelength $\lambda$. The spectrum and amplitude of $\sigma_{\text {ext }}$ can thus be determined. ${ }^{[2,24]}$ The broadly tunable source required for measurement of the spectra was provided by a femtosecond Ti:Sapphire oscillator (pulse duration $\approx 150 \mathrm{fs}$ ) associated with a femtosecond optical parametric oscillator (OPO) working in the visible. Using either the oscillator or the OPO, a light source tunable in the $490-1040 \mathrm{~nm}$ range was obtained. Beam focusing was achieved by a microscope objective (100x magnification, 0.75 numerical aperture), yielding a focal spot size of $\approx 0.7 \lambda$ (full width at half maximum of the light intensity profile) close to the diffraction limit. The sample position was modulated at $f \approx 1.5 \mathrm{kHz}$ and the transmitted light power detected by a photodiode, its $2 f$ component being extracted by a lock-in amplifier. Light polarization was controlled using the combination of a quarterwave plate and a polarizer. For all the investigations reported here, it was rotated to maximize extinction at the longitudinal SPR.

Electron Tomography (3D TEM): Acquisitions were performed on a JEOL $2100 \mathrm{~F}$ transmission electron microscope with a field emission gun and a post-column GATAN Tridiem energy filter. The tilt series have been recorded in an automatic manner using the GATAN tomography software. In the bright field mode (for the Au bipyramid), the tilt series was recorded by covering an angular range between $-70^{\circ}$ an $+70^{\circ}$ with an increment of $2^{\circ}$ following the Saxton scheme. The TEM images are made of $2048 \times 2048$ pixels with a pixel size of $0.16 \mathrm{~nm}$. No visible beam damage was observed after the acquisition. For the HAADF-STEM (in the case of Au@Ag core-shell bipyramid), the tilt series was acquired using the ADF detector by tilting the specimen in the angular range of $\pm 70^{\circ}$ with an increment of $2.5^{\circ}$ in the equal mode, thus giving a total number of images of 60 . The large inner radius chosen for the ADF detector, $40 \mathrm{mrad}$, ensures a good proportionality between the intensity of images and the mean atomic number. The gain of the ADF detector was chosen to prevent saturation effects in the HAADF images during the tilt series acquisition.

\section{Supporting Information}

Supporting Information is available from the Wiley Online Library or from the author.

\section{Acknowledgement}

This work was supported by the Agence Nationale de la Recherche (ANR) under Contract ANR-BLAN-SIMI10-LS-100617-15-01. S. G.-G. acknowledges the LabEx AMADEus (ANR-10-LABX-42) in the framework of IdEx Bordeaux (ANR-10-IDEX-03-02).

[1] K. L. Kelly, E. Coronado, L. L. Zhao, G. C. Schatz, J. Phys. Chem. B 2003, 107, 668.

[2] A. Crut, P. Maioli, N. Del Fatti, F. Vallée, Chem. Soc. Rev. 2014, 43, 3921.
[3] J. N. Anker, W. P. Hall, O. Lyandres, N. C. Shah, J. Zhao, R. P. Van Duyne, Nat. Mater. 2008, 7, 442.

[4] M. A. Otte, B. Sepúlveda, W. Ni, J. P. Juste, L. M. Liz-Marzán, L. M. Lechuga, ACS Nano 2010, 4, 349.

[5] C. E. Talley, J. B. Jackson, C. Oubre, N. K. Grady, C. W. Hollars, S. M. Lane, T. R. Huser, P. Nordlander, N. J. Halas, Nano Lett. 2005, $5,1569$.

[6] B. Sharma, R. R. Frontiera, A.-I. Henry, E. Ringe, R. P. Van Duyne, Mater. Today 2012, 15, 16.

[7] S. Khatua, P. M. R. Paulo, H. Yuan, A. Gupta, P. Zijlstra, M. Orrit, ACS Nano 2014, 8, 4440.

[8] E. M. Larsson, J. Alegret, M. Käll, D. S. Sutherland, Nano Lett. 2007, 7, 1256

[9] S. Lal, S. Link, N. J. Halas, Nat. Photonics 2007, 1, 641.

[10] K. C. Vernon, A. M. Funston, C. Novo, D. E. Gómez, P. Mulvaney, T. J. Davis, Nano Lett. 2010, 10, 2080.

[11] L. J. Sherry, S.-H. Chang, G. C. Schatz, R. P. Van Duyne, B. J. Wiley, Y. Xia, Nano Lett. 2005, 5, 2034.

[12] M. W. Knight, Y. Wu, J. B. Lassiter, P. Nordlander, N. J. Halas, Nano Lett. 2009, 9, 2188.

[13] P. Albella, B. Garcia-Cueto, F. González, F. Moreno, P. C. Wu, T.-H. Kim, A. Brown, Y. Yang, H. O. Everitt, G. Videen, Nano Lett. 2011, 11, 3531.

[14] H. Chen, L. Shao, T. Ming, K. C. Woo, Y. C. Man, J. Wang, H.-Q. Lin, ACS Nano 2011, 5, 6754.

[15] A. Lombardi, M. Loumaigne, A. Crut, P. Maioli, N. Del Fatti, F. Vallée, M. Spuch-Calvar, J. Burgin, J. Majimel, M. TréguerDelapierre, Langmuir 2012, 28, 9027.

[16] J. J. Mock, R. T. Hill, A. Degiron, S. Zauscher, A. Chilkoti, D. R. Smith, Nano Lett. 2008, 8, 2245.

[17] D. Y. Lei, A. I. Fernández-Domínguez, Y. Sonnefraud, K. Appavoo, R. F. Haglund, J. B. Pendry, S. A. Maier, ACS Nano 2012, 6, 1380.

[18] F. Le, N. Z. Lwin, J. M. Steele, M. Käll, N. J. Halas, P. Nordlander, Nano Lett. 2005, 5, 2009.

[19] T. G. Habteyes, S. Dhuey, E. Wood, D. Gargas, S. Cabrini, P. J. Schuck, A. P. Alivisatos, S. R. Leone, ACS Nano 2012, 6, 5702.

[20] T. Siegfried, Y. Ekinci, O. J. F. Martin, H. Sigg, ACS Nano 2013, 7, 2751.

[21] A. Hoggard, L.-Y. Wang, L. Ma, Y. Fang, G. You, J. Olson, Z. Liu, W.-S. Chang, P. M. Ajayan, S. Link, ACS Nano 2013, 7, 11209.

[22] P. A. Midgley, M. Weyland, Ultramicroscopy 2003, 96, 413.

[23] B. Nikoobakht, M. A. El-Sayed, Chem. Mater. 2003, 15, 1957.

[24] A. Arbouet, D. Christofilos, N. Del Fatti, F. Vallée, J. Huntzinger, L. Arnaud, P. Billaud, M. Broyer, Phys. Rev. Lett. 2004, 93, 127401.

[25] O. Muskens, D. Christofilos, N. Del Fatti, F. Vallée, J. Opt. A Pure Appl. Opt. 2006, 8, S264.

[26] D. Christofilos, J.-C. Blancon, J. Arvanitidis, A. San Miguel, A. Ayari, N. Del Fatti, F. Vallée, J. Phys. Chem. Lett. 2012, 3, 1176.

[27] V. Juvé, M. F. Cardinal, A. Lombardi, A. Crut, P. Maioli, J. Pérez-Juste, L. M. Liz-Marzán, N. Del Fatti, F. Vallée, Nano Lett. 2013, 13, 2234.

[28] C. Sönnichsen, T. Franzl, T. Wilk, G. von Plessen, J. Feldmann, O. Wilson, P. Mulvaney, Phys. Rev. Lett. 2002, 88, 077402.

[29] U. Kreibig, Appl. Phys. B 2008, 93, 79.

[30] M. Hu, C. Novo, A. Funston, H. Wang, H. Staleva, S. Zou, P. Mulvaney, Y. Xia, G. V. Hartland, J. Mater. Chem. 2008, 18, 1949.

[31] H. Baida, P. Billaud, S. Marhaba, D. Christofilos, E. Cottancin, A. Crut, J. Lermé, P. Maioli, M. Pellarin, M. Broyer, N. Del Fatti, F. Vallée, A. Sánchez-Iglesias, I. Pastoriza-Santos, L. M. Liz-Marzán, Nano Lett. 2009, 9, 3463.

[32] P. Zijlstra, P. M. R. Paulo, K. Yu, Q.-H. Xu, M. Orrit, Angew. Chem. Int. Ed. 2012, 51, 8352.

[33] J. Lermé, H. Baida, C. Bonnet, M. Broyer, E. Cottancin, A. Crut, P. Maioli, N. Del Fatti, F. Vallée, M. Pellarin, J. Phys. Chem. Lett. 2010, 1, 2922. 
[34] Y. R. Davletshin, A. Lombardi, M. F. Cardinal, V. Juvé, A. Crut, P. Maioli, L. M. Liz-Marzán, F. Vallée, N. Del Fatti, J. C. Kumaradas, ACS Nano 2012, 6, 8183.

[35] O. L. Muskens, G. Bachelier, N. Del Fatti, F. Vallée, A. Brioude, X. Jiang, M.-P. Pileni, J. Phys. Chem. C 2008, 112, 8917.

[36] H. Baida, D. Christofilos, P. Maioli, A. Crut, N. Del Fatti, F. Vallée, in Proc. SPIE 7033, Plasmon. Nanoimaging, Nanofabrication, Their Appl. IV (Eds: S. Kawata, V. M. Shalaev, D. P. Tsai), 2008, p. 703319.

[37] C. F. Bohren, D. P. Gilra, J. Colloid Interface Sci. 1979, 72, 215.

[38] A. N. Lebedev, M. Gartz, U. Kreibig, O. Stenzel, Eur. Phys. J. D 1999, 6,365 .

[39] W. W. Duley, Astrophys. J. 1984, 287, 694.

[40] N. Savvides, J. Appl. Phys. 1986, 59, 4133.

[41] B. Mednikarov, G. Spasov, T. Babeva, J. Pirov, M. Sahatchieva, C. Popov, W. Kulisch, J. Optoelectron. Adv. Mater. 2005, 7, 1407.

[42] P. B. Johnson, R. W. Christy, Phys. Rev. B 1972, 6, 4370.

[43] E. C. Le Ru, J. Grand, I. Sow, W. R. C. Somerville, P. G. Etchegoin, M. Treguer-Delapierre, G. Charron, N. Félidj, G. Lévi, J. Aubard, Nano Lett. 2011, 11, 5013.
[44] D.-S. Kim, J. Heo, S.-H. Ahn, S. W. Han, W. S. Yun, Z. H. Kim, Nano Lett. 2009, 9, 3619.

[45] J. Burgin, I. Florea, J. Majimel, A. Dobri, O. Ersen, M. TréguerDelapierre, Nanoscale 2012, 4, 1299.

[46] O. Ersen, I. Florea, C. Hirlimann, C. Pham-Huu, Mater. Today 2015, 18, 395.

[47] J. Burgin, M. Liu, P. Guyot-Sionnest, J. Phys. Chem. C 2008, 112, 19279.

[48] M. Liu, P. Guyot-Sionnest, J. Phys. Chem. B 2004, 108, 5882.

[49] J. Becker, I. Zins, A. Jakab, Y. Khalavka, O. Schubert, C. Sönnichsen, Nano Lett. 2008, 8, 1719.

[50] A. Lombardi, M. P. Grzelczak, A. Crut, P. Maioli, I. PastorizaSantos, L. M. Liz-Marzán, N. Del Fatti, F. Vallée, ACS Nano 2013, 7, 2522.

[51] M. Liu, P. Guyot-Sionnest, J. Phys. Chem. B 2005, 109, 22192.

[52] E. C. Le Ru, J. Grand, I. Sow, W. R. C. Somerville, P. G. Etchegoin, M. Treguer-Delapierre, G. Charron, N. Félidj, G. Lévi, J. Aubard, Nano Lett. 2011, 11, 5013. 